\title{
The Role of the Robot Mass and Velocity in Physical Human-Robot Interaction - Part II: Constrained Blunt Impacts
}

\author{
Sami Haddadin, Alin Albu-Schäffer, Mirko Frommberger, and Gerd Hirzinger
}

\begin{abstract}
Accidents occurring with classical industrial robots often lead to fatal injuries. Presumably, this is to a great extent caused by the possibility of clamping the human in the confined workspace of the robot. Before generally allowing physical cooperation of humans and robots in future applications it is therefore absolutely crucial to analyze this extremely dangerous situation. In this paper we will investigate many aspects relevant to this sort of injury mechanisms and discuss the importance to domestic environments or production assistants. Since clamped impacts are intrinsically more dangerous than free ones it is fundamental to discuss and evaluate metrics to ensure safe interaction if clamping is possible. We compare various robots with respect to their injury potential leading to a main safety requirement of robot design: Reduce the intrinsic injury potential of a robot by reducing its weight.
\end{abstract}

\section{Motivation \& InTRODUCTION}

In the first part of this work [1] non-constrained blunt impacts were investigated with respect to robot mass and velocity. The effect of these robot parameters in case of clamping will be outlined in this second part. Robotics literature deals mainly with free impacts [2], [3], [4], only few works as e.g. [5] give a short notion about the injury potential emanating from clamping.

In part I we showed that the intuition of a massive robot being much more dangerous than a light one, or being even life threatening does not generally apply. A saturation of potential injury observed for free impacts even led to the conclusion that no robot whatever mass it has can become life threatening at typical robot speeds ${ }^{1}$ if the human is not clamped $^{2}$. In this paper we will point out that in case of a clamped human intuition turns out to be correct, meaning that the heavier the robot is, the more severe injuries are likely to occur.

Concerning injuries caused by robots, only very little data or literature is available. In [6] the United Auto Workers (UAW) union published a report which provides raw data on various injuries related to robot operations. It indicates that a major fraction of occurring injuries involve somehow clamping of a human body part. Since it is not feasible to adequately treat all different contact types in this paper,

S. Haddadin, A. Albu-Schäffer, Mirko Frommberger and G. Hirzinger are with Institute of Robotics and Mechatronics, DLR German Aerospace Center, Wessling, Germany sami.haddadin, alin.albu-schaeffer, mirko.frommberger, gerd.hirzingeredlr.de

This work has been partially funded by the European Commission's Sixth Framework Programme as part of the projects SMERobot ${ }^{\mathrm{TM}}$ under grant no. 011838 and PHRIENDS under grant no. 045359.

${ }^{1}$ There are exceptions as e.g. painting robots which move with up to $8 \mathrm{~m} / \mathrm{s}$.

${ }^{2}$ With respect to typical severity indices used in the automobile industry. However, in this paper we investigated fractures which are partially classified as minor injuries. Nevertheless, the question arises which secondary injuries can be the consequence. we will concentrate on blunt contact. Of course in reality different contact areas for various tools and structural edges are possible and can/should be incorporated in future work ${ }^{3}$. A typical situation where a human operator can be clamped is e.g. during maintenance of a robotic work cell. Due to the (partially) confined workspace it is possible to get clamped e.g. between the safety fence or a workbench and the robotic structure ${ }^{4}$. In order to analyze the mechanisms behind such a process we first explain which types of blunt clamping are relevant to robotics and next we give the braking distance of various investigated robots. These tests are especially done for estimating the equivalent braking force for a one-dimensional impact simulation ${ }^{5}$ which will be used to analyze maximal contact forces and evaluate severity indices. This is necessary to analyze constrained impacts with biomechanical models of the human head and chest. We have to rely on these validated simulations because unfortunately, real clamping tests with a crash-test dummy (e.g. HIII) are not realizable without destroying the equipment. In these simulations it is assumed that the robot is able to detect a collision and immediately engages its brakes. It seems clear to us that (at least) an industrial robot is able to generate forces high enough to kill a clamped human if it is not able to react at all and just continues to follow its desired trajectory.

Furthermore, we show that with a robot like the DLRLightweight Robot III (LWRIII), which is especially designed for human-robot interaction, clamping is under normal circumstances not leading to life-threatening injury by means of typical injury measures in the automobile industry, but less severe injuries like fractures of facial and cranial bones can occur ${ }^{6}$.

The paper is organized as follows. Sec. II explains the two major types of clamping, followed by Sec. III, describing the braking tests with the LWRIII and three different industrial robots. Sec. IV shows some results obtained by clamping tests with the LWRIII and the KUKA KR6. To draw some general statements clamping simulations were carried out in Sec. V and finally a conclusion and outlook are given in Sec. VI.

\section{TyPeS OF BLUNT CLAMPING}

Generally, two types of blunt clamping can be differentiated: Dynamic and quasistatic. According to [6] the first one

\footnotetext{
${ }^{3} \mathrm{~A}$ paper discussing these issues is currently in preparation.

${ }^{4}$ Clamping can as well occur within robotic elements, such as two links, but this is not part of our analysis.

${ }^{5} \mathrm{~A}$ full dynamic model of the industrial robots for simulation is not available.

${ }^{6}$ This does not mean these are the only possible injuries, other ones like contre coup [7] or secondary injuries need further investigation.
} 

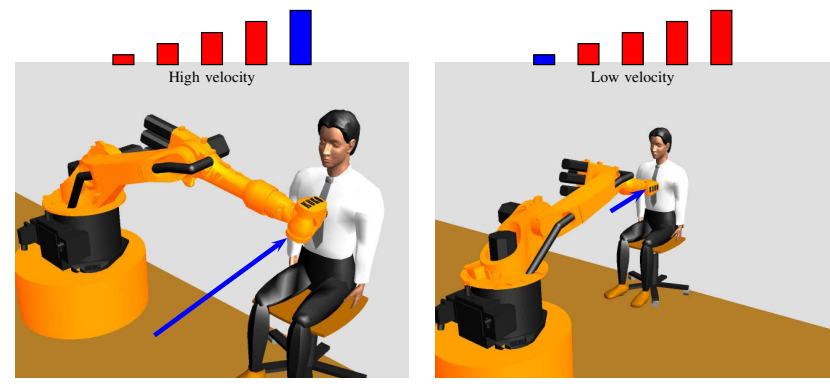

Fig. 1. Two different types of clamping: Dynamic clamping at high Cartesian velocities (left) and quasistatic clamping during low velocity movements or near singularities (eventually high joint velocities but slow Cartesian velocity).

is a major injury source in industrial applications and will be the focus of this paper. The second one occurs if the robot is moving very slowly or if the robot is close to a singularity. It is discussed to some extent in [8].

- Dynamic Clamping: Dynamic clamping describes the situation where the human is trapped against a rigid object while the robot moves at considerably high Cartesian velocities and hits the human body part as e.g. indicated in Fig. 1 (left).

- Quasistatic Clamping: The injury potential of a quasistatic collision is mainly defined by the maximum force the robot is able to exert and the space available to crush the body $\operatorname{part}^{7}$ as indicated in Fig. 1 (right).

\section{BRAKING TESTS}

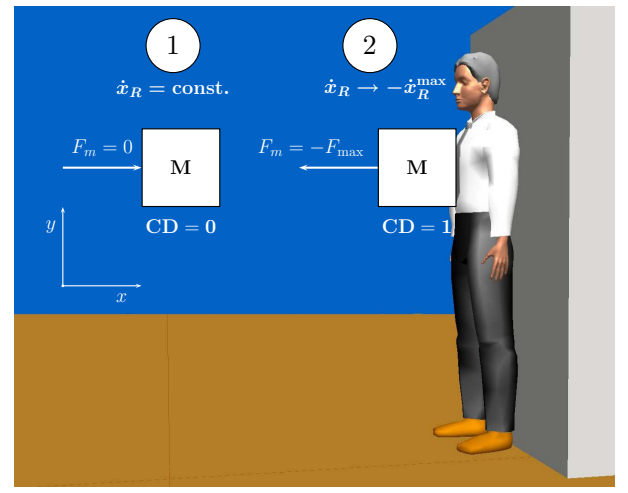

Fig. 2. Reduced clamping model for the industrial robots. $C D$ denotes the binary collision detection signal. The robot is assumed to approach with constant velocity and as soon as a collision is detected exerts the maximum braking force on the robot inertia reflected at the tip in moving direction until contact with the clamped human is lost.

The braking distance was measured at various initial velocities, serving especially two purposes:

1) Obtain and compare measurements of the braking distances of real robots at typical velocities.

\footnotetext{
${ }^{7}$ The space available describes whether enough distance is available with respect to the robot's workspace in order to exceed the particular tolerance values of the body part.
}

2) Calculate the equivalent braking force for a reduced one-dimensional model of the particular industrial robot.

The one-dimensional model contains the relevant Cartesian direction of the reflected robot mass $\mathbf{M}_{c} \in \Re^{6 \times 6}$ at the tip [9]

$$
\mathbf{M}_{c}=\left(\mathbf{J}(\mathbf{q}) \mathbf{M}(\mathbf{q})^{-1} \mathbf{J}(\mathbf{q})^{T}\right)^{-1}
$$

where $\boldsymbol{q} \in \Re^{n}$ is the link position of the robot, $\mathbf{M}(\mathbf{q}) \in$ $\Re^{n \times n}$ is the link mass matrix, and $\mathbf{J}(\mathbf{q}) \in \Re^{6 \times n}$ the manipulator Jacobian.

In order to measure the braking distance of the robots (except for the LWRIII) they were abruptly stopped at various (up to full) speeds with and without brakes during their commanded trajectory execution ${ }^{8}$. In this paper we will use the braking distance, obtained for a particular configuration and velocity, to simulate impacts with clamped humans. In Fig. 2 the desired model is shown: The robot is represented by its reflected Cartesian inertia, listed in [1], and moves at constant velocity (1). As soon as a collision is detected the robot immediately brakes with maximum available force (2). The braking force acting on the reflected inertia will be estimated from the real trajectories, see Appendix. All models used for the head and chest of the human can be found in [10], [11], [12], [13], [14].

Tab. I compares the braking distance and time of all evaluated

\begin{tabular}{|c|c|c|c|c|}
\hline Robot & $\Delta t_{\text {idle }}[\mathrm{ms}]$ & $\Delta t_{\text {stop }}[\mathrm{ms}]$ & $\Delta x_{R}[\mathrm{~cm}]$ & $\Delta x_{\text {idle }}[\mathrm{cm}]$ \\
\hline LWRIII (link) & $11-23$ & 200 & $0.55-6.8$ & $0.23-4.8$ \\
\hline LWRIII (dummy) & $11-23$ & 200 & $0.25-4.2$ & $0.23-4.8$ \\
\hline LWRIII (motor) & 4 & 250 & not def. & not.def. \\
\hline KR3-SI (Cat.0) & $36-48$ & $200-300$ & $6.5-34$ & $2.6-9.6$ \\
\hline KR6 (Cat.1) & $36-48$ & $150-200$ & $6-24$ & $2.4-9.5$ \\
\hline KR6 (Cat.0) & 36 & $48-132$ & $1-17$ & $0.8-7$ \\
\hline KR500 (Cat.1) & $60-72$ & $400-650$ & $16-69$ & $4.2-14$ \\
\hline KR500 (Cat.0) & $12-24$ & $60-336$ & $0.8-42$ & $0.6-7$ \\
\hline $\mathrm{KR} 6_{\dot{q}_{1}^{\max }}$ (Cat.1) & 36 & 252 & 55 & 13 \\
\hline $\mathrm{KR}_{\dot{q}_{1}^{\max }}($ Cat.0) & 36 & 216 & 45 & 13 \\
\hline $\mathrm{KR} 500_{\dot{q}_{1}^{\max }}$ (Cat.1) & 85 & 1000 & 186 & 26 \\
\hline $\mathrm{KR} 500_{\dot{q}_{1}^{\max }}$ (Cat.0) & 36 & 564 & 121 & 13 \\
\hline
\end{tabular}

TABLE I

COMPARISON OF CARTESIAN BRAKING DISTANCES AND TIME FOR IMPACT VELOCITIES OF $0.2-2 \mathrm{~m} / \mathrm{s}$ FOR ALL ROBOTS. FOR THE LWRIII AN IMPACT REDUCES THE BRAKING DISTANCE SIGNIFICANTLY (SHADED GREY). BRAKING CHARACTERISTICS FOR MAXIMUM VELOCITIES (SHADED RED) OF KR6 AND KR500.

robots $^{9}$. It clearly shows that increasing the robot mass results in very large braking distances up to $690 \mathrm{~mm}$ for

\footnotetext{
${ }^{8}$ Further work is still necessary to accurately determine the braking distance of a robot in arbitrary states.

${ }^{9}$ We compared the LWRIII with the KUKA KR3 $(54 \mathrm{~kg})$, the KUKA
} KR6 (235 kg), and the KUKA KR500 (2350 kg). 
the KR500 at robot speeds up to $2 \mathrm{~m} / \mathrm{s}$ at Category 1 stop $^{10}$. At maximum joint velocity $(3.7 \mathrm{~m} / \mathrm{s}$ Cartesian velocity) the KR500 needs almost $2 \mathrm{~m}$ at Category 1 to fully stop, see Fig. 12. Category 1 stops significantly reduce the braking distance. Furthermore, a comparison concerning idle and stop time $\left(\Delta t_{\text {stop }}=\Delta t_{\text {idle }}+\Delta t_{\text {brake }}\right)$ and idle and stop distance is given in Tab. I which already suggests the assumption that collisions could become fatal in case of clamping. Detailed plots of these experiments are given in the Appendix.

\section{Test WITH THE LWRIII \& KR6}

Before analyzing clamping in simulation two interesting experiments will be investigated.

\section{A. Impacting a Crash-test Dummy}
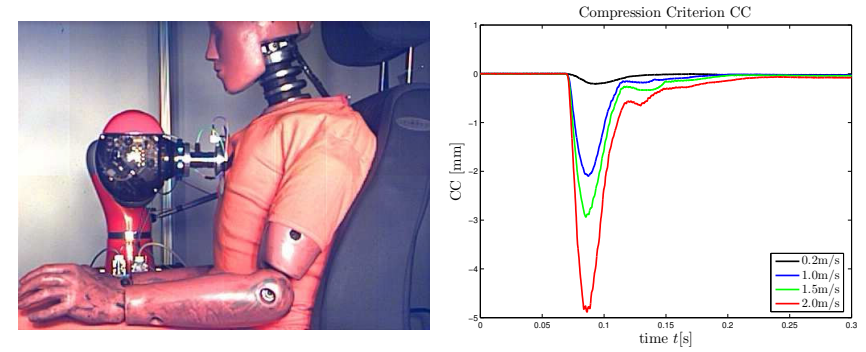

Fig. 3. Impact tests with a clamped HIII. The robot hits the dummy in outstretched configuration at various impact velocities (left). Measured Compression Criterion for a clamped HIII with the LWRIII. All values correlate to very low possible injury by means of the EuroNCAP (right).

In Fig. 3 (left) an impact of the LWRIII with a Hybrid III Dummy (HIII) sitting in (and at the same time confined by) a car seat is shown. For all impact velocities the maximum nominal joint torques are exceeded and consequently the robot stops. Alternatively, in case the collision detection [15] is activated the robot reacts compliantly since the reaction scheme is able to limit the joint torques and prevents the previously mentioned low-level stop. This is possible ${ }^{11}$ up to impact velocities of almost $2 \mathrm{~m} / \mathrm{s}$. From the high-speed videos we recorded at a framerate of $1 \mathrm{kHz}$ it is clearly observed that the actual impact is completely over before the trunk of the dummy starts moving and gets pushed into the seat. Therefore, the compliance of the seat did not influence the impact. In other words, the chest impact dynamics do not differ for the LWRIII, no matter whether the dummy is clamped or not. In Fig. 3 (right) one can see the resulting Compression Criterion ${ }^{12}$ (CC) plots for various impact velocities $\left\|\dot{\mathbf{x}}_{R}\right\| \in\{0.2,1.0,1.5,2.0\} \mathrm{m} / \mathrm{s}$. The maximal numerical value of $5 \mathrm{~mm}$ is far below the threshold value of $22 \mathrm{~mm}$ corresponding to very low injury by means of EuroNCAP ${ }^{13}$. Therefore, no serious injury of

\footnotetext{
${ }^{10}$ The stop category is defined in DIN EN 60204. Category 0 stop means that the drives are immediately switched off and the brakes engage at the same time. A Category 1 stop lets the robot halt with a hard stop trajectory without using the brakes.

${ }^{11}$ In contrast to the significantly harder impact with the head, where the collision detection and reaction cannot contribute to the reduction of joint torques anymore already at moderate robot speed [16].

${ }^{12}$ The used severity indices are introduced and explained in [1].

${ }^{13}$ EuroNCAP provides a rating of injury potential for automobile crashtesting: [very low injury $\equiv$ green] to [very high injury $\equiv$ red]. For details on EuroNCAP please refer to [16].
}

the chest can occur with the LWRIII if the human is clamped because the maximal nominal joint torques are exceeded before the $\mathrm{CC}$ values could become critical. This is true, even if the collision detection fails.

\section{B. Cracking a Coconut}
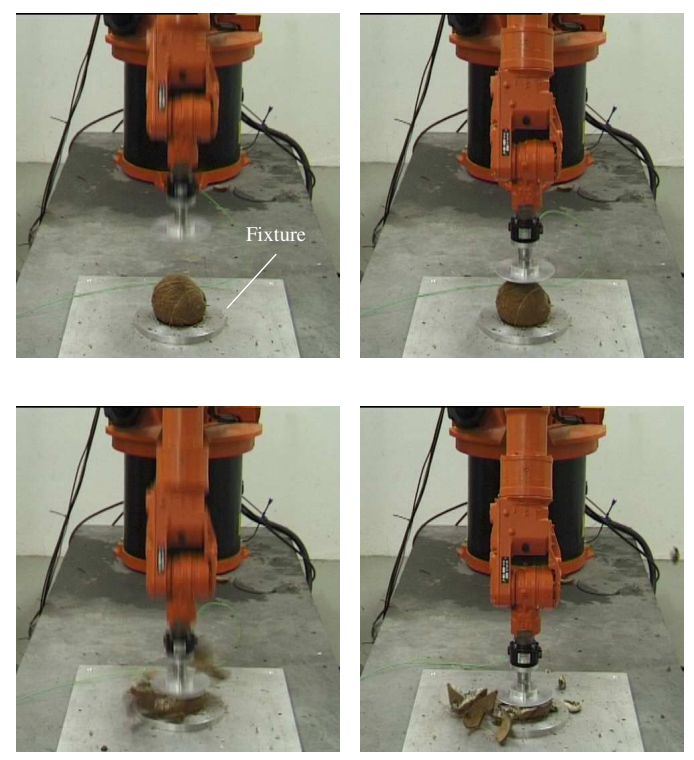

Fig. 4. Cracking a coconut with a KR6. An aluminum fixture keeps it centered.
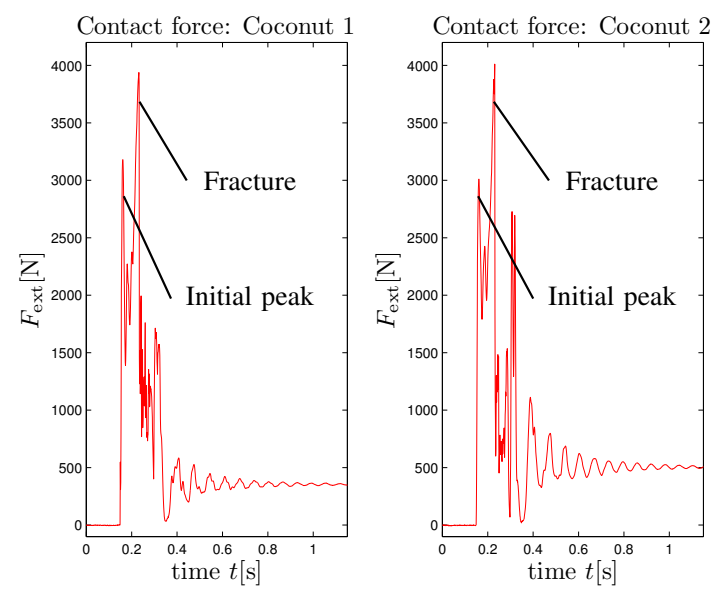

Fig. 5. Cracking a coconut with the KR6. Contact force profiles for two different sample coconuts.

A major drawback of crash-test dummies is that they cannot be used to measure forces acting on the clamped head. In order to illustrate the threat emanating from heavy hightorque robots we decided to show what a $6 \mathrm{~kg}$-payload robot like the KR6 is already capable of via an intuitive example: Cracking a clamped coconut, see Fig. 4. The robot moves on a predefined trajectory in Cartesian space and impacts the coconut with $0.6 \mathrm{~m} / \mathrm{s}$. The coconut is not able to slip away due to an aluminum fixture keeping it centered. The force needed to crack the nut with the blunt impactor is $F_{\text {ext }} \approx$ $4 \mathrm{kN}$, as indicated in Fig. 5 by the force profiles of such cracks for two different coconuts. It is not perfectly clear, whether the initial smaller peak is due to the dynamics of 
the impact (robot, controller, contact dynamics), slippage or a first partial crack in the structure. However, slippage seems very unlikely due to the reproducibility of the experiment, the fixture and the high stiffness of the robot. An initial crack is less probable as well due to the smooth behavior after the initial peak. The measured fracture force corresponds to the typical one of the human frontal bone [1]. The magnitude of the required fracture force shows that this experiment is a sufficient showcase for the clamping of a human head, which would be similarly battered ${ }^{14}$. In the next section impact simulations are going to be examined, leading to some more general statements.

\section{Simulations}

In this section the results of the impact simulations with a full model of the LWRIII and one-dimensional representations of the industrial robots are shown. Again, the collision detection and reaction strategy for the LWRIII are the ones described in [15]. The reflected inertias of the industrial robots and the description of fracture forces and severity indices can be found in [1].

\section{A. Facial Impact Forces with Clamping}

\begin{tabular}{lcc}
\hline ROBOT & Contact Force & Maxilla Fracture? \\
\hline LWRIII & $0.6 \mathrm{kN} @ 1 \mathrm{~m} / \mathrm{s}$ & No \\
LWRIII & $1.2 \mathrm{kN} @ 2 \mathrm{~m} / \mathrm{s}$ & Yes \\
KR3 & $2.2 \mathrm{kN} 2 \mathrm{~m} / \mathrm{s}$ & Yes \\
KR6 (Cat.0\&1) & $5.1 \mathrm{kN} 2 \mathrm{~m} / \mathrm{s}$ & Yes \\
KR500 (Cat.0\&1) & $23.6 \mathrm{kN} @ 2 \mathrm{~m} / \mathrm{s}$ & Yes \\
\hline ROBOT & Contact Force & Frontal Fracture? \\
\hline LWRIII & $3.5 \mathrm{kN} @ 2 \mathrm{~m} / \mathrm{s}$ & No \\
KR3 & $6.9 \mathrm{kN} @ 2 \mathrm{~m} / \mathrm{s}$ & Yes \\
KR6 (Cat.0\&1) & $16.3 \mathrm{kN} @ 2 \mathrm{~m} / \mathrm{s}$ & Yes \\
KR500 (Cat.0\&1) & $86.3 \mathrm{kN} @ 2 \mathrm{~m} / \mathrm{s}$ & Yes \\
\hline
\end{tabular}

TABLE II

CONSERVATIVE IMPACT FORCES WITH CLAMPING AT $2 \mathrm{~m} / \mathrm{s}$ OBTAINED FOR THE MAXILLA AND FRONTAL BONE.

In Tab. II the clamping forces of the maxilla and frontal bone ${ }^{15}$ for impacts at $2 \mathrm{~m} / \mathrm{s}$ for all robots ${ }^{16}$ in their particular impact configuration are listed. The robot reacts to the collision by braking with maximum reverse torque and continuing until contact with the head is lost. The simulations show the vast influence of the relation robot mass $\leftrightarrow$ braking or motor torque and already the KR3 produces twice the contact force the LWRIII generates ${ }^{17}$. However, all robots potentially break

\footnotetext{
${ }^{14}$ See also the attached video. However, according to [17] a human head would usually slip away for quasi-static loading. This was observed as well for the coconut, leading to the usage of the aluminum fixture.

${ }^{15}$ Other bones were investigated as well, but their analysis would not contribute additional insight.

${ }^{16}$ For this simulation the KR3-SI is assumed to have no intermediate flange with breakaway function, i.e. we assume a KR3.

${ }^{17}$ The relation between motor torque and inertia scales disadvantageously when increasing dimensions.
}

the maxilla and even the low inertia LWRIII threatens this particular bone at $2 \mathrm{~m} / \mathrm{s}$. Nonetheless, one should keep in mind that the model and fracture forces assumed in this simulation are kept very conservative. The linear model assumption does e.g. not take into account an initial sublinear characteristic of the real force-deflection relationship of the bone [12], [13]. Furthermore, are the fracture forces used in [1] very conservative ones found in the literature. For the LWRIII the resulting maximal allowable velocity is $\approx 1 \mathrm{~m} / \mathrm{s}$ for maxilla impacts if the stop is performed without brakes. With brakes this critical velocity could be significantly higher due to the reduced braking distance, see Appendix Fig. 9. For the frontal bone even $2 \mathrm{~m} / \mathrm{s}$ is still a safe velocity in case of the LWRIII. For industrial robots a difference between Cat. 0 and 1 stop cannot be observed, showing the inherent danger emanating from such heavy robots (for both evaluated bones). However, not only the force should be considered but the deflection as well. For the KR500 a numerical value of $236 \mathrm{~mm}$ is obtained for the maxilla, which is of course deadly. Additionally, one has to take into consideration that the applied human model is not valid anymore after the fracture occurs. This is because the resistance of the human head is dramatically lowered, possibly causing even more severe injury (higher deflections after the fracture will occur and lead to numerous internal injuries).

\section{B. Chest Impacts with Clamping}

\begin{tabular}{lrrr}
\hline ROBOT & $\mathrm{CC}[\mathrm{mm}]$ & $\mathrm{VC}[\mathrm{m} / \mathrm{s}]$ & $F_{\text {ext }}^{x}[\mathrm{~N}]$ \\
\hline LWRIII & $14.4(0.0)$ & 0.035 & $741.6(1.3)$ \\
KR3 (Cat.0) & $31.2(0.0)$ & 0.1 & $851.9(1.4)$ \\
KR6 (Cat.0) & $65.5(2.0)$ & 0.25 & $2836.1(2.7)$ \\
KR6 (Cat.1) & $66.6(2.1)$ & 0.25 & $2904.6(2.7)$ \\
KR500 (Cat.0) & $228.0(6.0)$ & 0.84 & $14282.0(6.0)$ \\
KR500 (Cat.1) & $245.0(6.0)$ & 0.89 & $15491.0(6.0)$ \\
\hline
\end{tabular}

TABLE III

SIMULATED VALUES FOR CHEST SEVERITY INDICES AND CORRESPONDING AIS VALUES AT $2 \mathrm{~m} / \mathrm{s}$ OBTAINED FOR THE HUMAN CHEST.

In Tab. III the Compression Criterion (CC), Viscous Criterion (VC) and the clamping force $F_{\text {ext }}^{x}$ of the chest are listed for all robots at $2 \mathrm{~m} / \mathrm{s}$ impact velocity. The corresponding EuroNCAP injury level is indicated for CC and VC. For the CC the AIS level ${ }^{18}$, obtained by the mappings introduced in [1], is additionally given in brackets as well. The contact force $F_{\text {ext }}^{x}$ is not part of the EuroNCAP evaluation but the corresponding AIS values according to [18] are denoted. The injury level of the $\mathrm{CC}$ and $F_{\text {ext }}^{x}$ show how increasing robot mass leads to a higher probability of injury level with respect to the EuroNCAP definition and/or AIS. The LWRIII does not pose a threat to the human chest, as already indicated in

\footnotetext{
${ }^{18}$ The Abbreviated Injury Scale (AIS) subdivides observed levels of injury into seven categories from $[$ none $\equiv 0]$ to $[$ fatal $\equiv 6]$. For details on AIS please refer to [16].
} 
Sec. IV-A. The KR6 on the other hand can cause very high injury by means of the EuroNCAP classification. The AIS mapping which is less conservative indicates approximately AIS $=2$, meaning recoverable injury. The KR500 is of course deadly as intuition already tells. The Viscous Criterion is due to the still quite low velocities subcritical except for the KR500 because the deflection then dominates the criterion ${ }^{19}$. The same conclusions as for the $\mathrm{CC}$ can be drawn from the contact force and its correlating injury level.

Similar to the head we can sum up that the chest is posed to a continously increasing threat with growing robot mass if the human is clamped. CC and $F_{\text {ext }}$ seem to be good indicators of injury for the chest in case of clamping due to their sensitivity in the relevant ranges.

In Fig. 6 the full time courses for $\mathrm{CC}$ and $\mathrm{VC}$ are given.
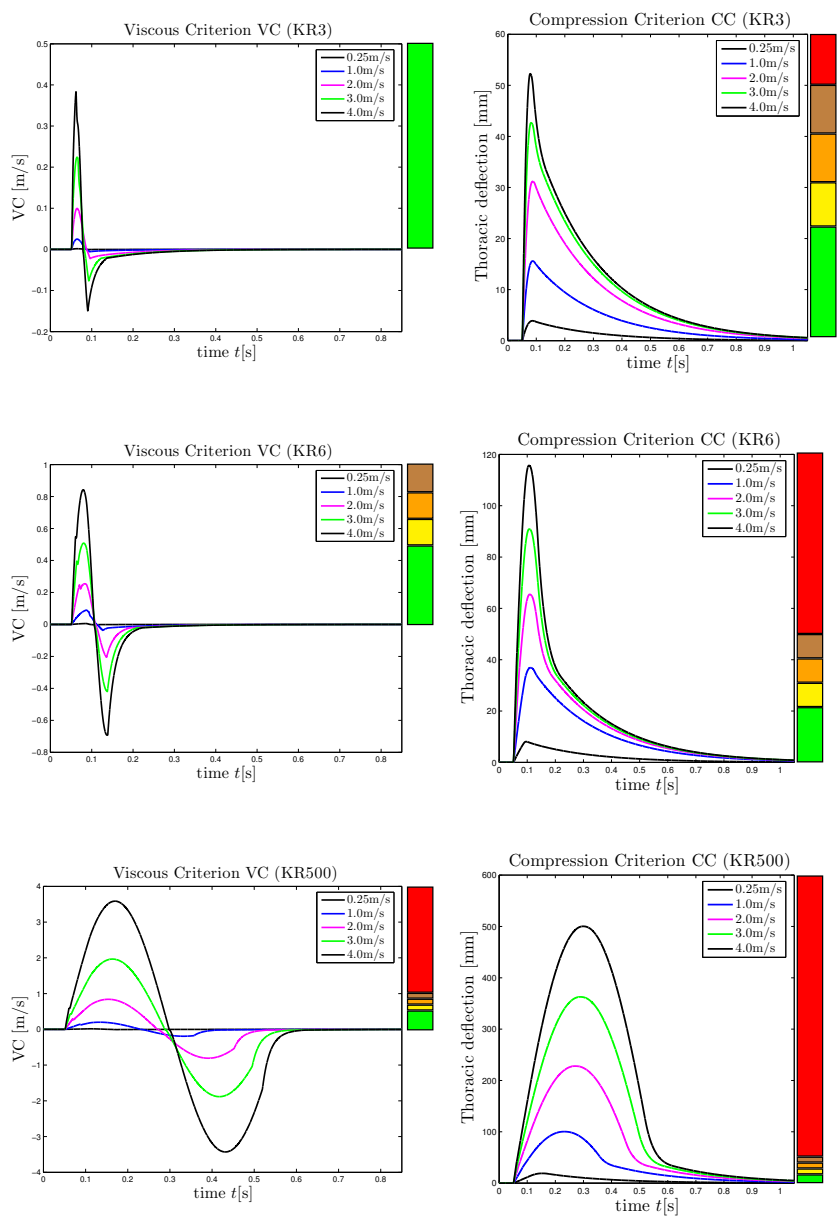

Fig. 6. Time courses of severity indices for simulated robot-chest collisions at various impact velocities with a clamped human chest for the KUKA KR3, KR6 \& KR500. The left column shows the time evolution of the Viscous Criterion and the right column the same one for the Compression Criterion. The colors indicate the injury potential with respect to EuroNCAP.

The left column shows the time evolution of the Viscous Criterion parameterized by impact velocities up to $4 \mathrm{~m} / \mathrm{s}$ and the right column the same for the Compression Criterion. The corresponding injury potential is indicated by the colored EuroNCAP bars. The Compression Criterion is clearly the

\footnotetext{
${ }^{19}$ This is consistent with the fact that VC is used for high velocity injuries in automobile crash-testing.
}

more sensitive and appropriate criterion for this type of collision. For the KR3 a velocity of $2 \mathrm{~m} / \mathrm{s}$ exceeds the possibility to keep very low injury potential, whereas for the KR6 already less than $1 \mathrm{~m} / \mathrm{s}$ is enough to exceed this threshold. In case of the KR500 only very low speeds of less than $0.5 \mathrm{~m} / \mathrm{s}$ are keeping the robot below the very low injury threshold.

\section{CONCLUSION \& OUTLOOK}

In this paper it was analyzed under which circumstances the intuition [heavy robot $\equiv$ dangerous robot] is true. In contrast to non-constrained blunt impacts which are characterized by a general saturation of injury potential over robot mass, clamping is a very dangerous situation which needs careful consideration and is apparently worth to be analyzed in detail. This aspect seems to be of major importance to us, since one can think of various situations in domestic or unstructured environments in which it is very likely to get clamped.

To our knowledge clamping in the context of human-robot interaction was covered only marginally if at all up to now. Simulations and measurements, representing the first of this kind were carried out for various different robots, ranging from manipulators especially designed for physical human-robot interaction to heavy industrial robots. In case of clamping both, the head and chest can be severely injured, even leading to death for increasing robot mass.

$\mathrm{CC}$ (chest) and $F_{\text {ext }}$ (head and chest) are shown to be good indicators of injury for blunt impacts in case of clamping. Clearly the tests and simulations pointed out the necessity of lightweight manipulators for the use in domestic environments. If the robot is too heavy its speed cannot be significantly reduced by the interaction forces in case of collision, i.e. the human body is not able to absorb the kinetic energy of the robot anymore without suffering from possibly severe or even lethal injuries. Furthermore, it is important to notice that braking distances of lighter robots are generally shorter which makes navigation uncertainties less dangerous for autonomous robots. An emergency braking has the possibility to significantly influence the behavior of the robot in extremely short terms. In this paper we investigated
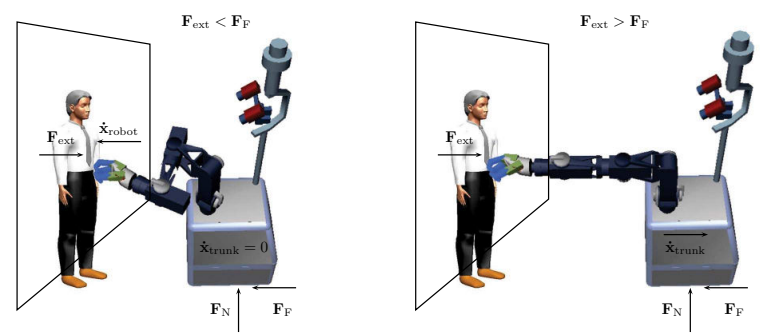

Fig. 7. Simplified and idealized visualization of clamping a human with a mobile manipulator. The trunk is assumed to stand still in the beginning and only the manipulator is moving towards the human body. The kinematic chain is assumed to be stiff and the torques caused by interaction are neglected.

robots with a fixed base, posing a risk especially for clamping because the robot cannot be entirely pushed away. For a mobile manipulator the situation is quite different: Especially in quasi-static clamping the friction forces of the mobile 
basis would define the maximum force the manipulator is able to exert on the clamped human, see Fig. 7. Therefore, the risk of clamping can be reduced, but on the other hand if the mobile platform is moving towards the human the overall speed and reflected inertia scale up. An analysis of a mobile manipulator is especially worthful for service robotics. Apart from classical mobile manipulators as [19], [20], [21] large humanoid upper body systems are mounted on a mobile platform [22], [23]. They introduce entirely new aspects to a consecutive safety analysis. Of course the outlined situation in Fig. 7 simplifies the problem and further need of investigation is apparent. However, interesting work discussing some of these issues were carried out in [5].

A video illustrating and supporting some key aspects proposed and explained in the paper is attached and further ones can be found at www.robotic.dlr.de/safe-robot.

\section{ACKNOWLEDGMENT}

We would like to thank Dr. Peter Heiligensetzer for carrying out the impact tests with the KUKA KR3-SI with us. Our special gratitude goes to Professor Dimitrios Kallieris for sharing his enormous biomechanical knowledge with us.

\section{REFERENCES}

[1] S. Haddadin, A. Albu-Schäffer, and G. Hirzinger, "The Role of the Robot Mass and Velocity in Physical Human-Robot Interaction - Part I: Unconstrained Blunt Impacts," in IEEE International Conference on Robotics and Automation (ICRA2008), Pasadena, USA, 2008.

[2] A. Bicchi and G. Tonietti, "Fast and Soft Arm Tactics: Dealing with the Safety-Performance Trade-Off in Robot Arms Design and Control," IEEE Robotics and Automation Mag., vol. 11, pp. 22-33, 2004.

[3] J. Heinzmann and A. Zelinsky, "Quantitative Safety Guarantees for Physical Human-Robot Interaction," Int. J. of Robotics Research, vol. 22, no. 7-8, pp. 479-504, 2003.

[4] M. Zinn, O. Khatib, and B. Roth, "A New Actuation Approach for Human Friendly Robot Design,” Int. J. of Robotics Research, vol. 23, pp. 379-398, 2004.

[5] H.-O. Lim and K. Tanie, "Human Safety Mechanisms of HumanFriendly Robots: Passive Viscoelastic Trunk and Passively Movable Base," Int. J. of Robotics Research, vol. 19, pp. 307-335, 2000.

[6] U. A. Workers, "Review of Robot Injuries - One of the Best Kept Secrets," in UAW Health and Safety Department Publication Nr.248.

[7] D. Kallieris, Handbuch gerichtliche Medizin, B. Brinkmann and B. Madea, Eds. Springer Verlag, 2004.

[8] S. Haddadin, A. Albu-Schäffer, and G. Hirzinger, "Safe Physical Human-Robot Interaction: Measurements, Analysis \& New Insights," in International Symposium on Robotics Research (ISRR2007), Hiroshima, Japan, 2007.

[9] O. Khatib, "Inertial properties in robotic manipulation: an object-level framework," Int. J. Robotics Research, vol. 14, no. 1, pp. 19-36, 1995.

[10] G. W. Nyquist, J. M. Cavanaugh, S. J. Goldberg, and A. I. King, "Facial Impact Tolerance and Response," SAE Paper No.861896, Proc. 30th Stapp Car Crash Conference, pp. 733-754, 1986.

[11] J. McElhaney, R. Stalnaker, and V. Roberts, "Biomechanical Aspects of Head Injury," Human Impact Response - Measurement and Simulation, 1972.

[12] D. L. Allsop, C. Y. Warner, M. G. Wille, D. C. Schneider, and A. M. Nahum, "Facial Impact Response-A Comparison of the Hybrid III Dummy and Human Cadaver," SAE Paper No.881719, Proc. 32th Stapp Car Crash Conference, pp. 781-797, 1988.

[13] D. L. Allsop and C. Y. Perl, Thomas R.and Warner, "Force/Deflection and Fracture Characteristics of the Temporo-parietal Region of the Human Head," SAE Transactions, pp. 2009-2018, 1991.

[14] T. Lobdell, C. Kroell, D. Scheider, and W. Hering, "Impact Response of the Human Thorax," Symposium on Human Impact Response, pp. 201-245, 1972.

[15] A. De Luca, A. Albu-Schäffer, S. Haddadin, and G. Hirzinger, "Collision Detection and Safe Reaction with the DLR-III Lightweight Manipulator Arm," IEEE/RSJ Int. Conf. on Intelligent Robots and Systems (IROS2006), Beijing, China, pp. 1623-1630, 2006.
[16] S. Haddadin, A. Albu-Schäffer, and G. Hirzinger, "Safety Evaluation of Physical Human-Robot Interaction via Crash-Testing," Robotics: Science and Systems Conference (RSS2007), Atlanta, USA, 2007.

[17] D. Kallieris, "Personal communication."

[18] C. Kroell, D. Scheider, and A. Nahum, "Impact Tolerance and Response of the Human Thorax II," SAE Paper No.741187, Proc. 18th Stapp Car Crash Conference, pp. 383-457, 1974.

[19] C. Ott, C. Borst, U. Hillenbrand, B. Brunner, B. Bäuml, and G. Hirzinger, "The Robutler: Towards Service Robots for the Human Environment," in VIDEO, IEEE Int. Conf. on Robotics and Automation (ICRA2005), Barcelona, Spain, 2005.

[20] R. Holmberg and O. Khatib, "Development and control of a holonomic mobile robot for mobile manipulation tasks." Int. J. of Robotics Research, vol. 19, no. 11, pp. 1066-1074, 2000.

[21] D. Katz, E. Horrell, Y. Yang, B. Burns, T. Buckley, A. Grishkan, V. Zhylkovskyy, O. Brock, and E. Learned-Miller, "The UMass Mobile Manipulator UMan: An Experimental Platform for Autonomous Mobile Manipulation."

[22] T. Asfour, K. Regenstein, P. Azad, J. Schröder, and R. Dillmann, "ARMAR-III: A Humanoid Platform for Perception-Action Integrationo," in Proceedings of the International Workshop on HumanCentered Robotic Systems (HCRS2006), Munich, Germany, 2006.

[23] J. Mehling, P. Strawser, L. Bridgwater, W. K. Verdeyen, and R. Rovekamp, "Centaur: NASA's Mobile Humanoid Designed for Field Work." in IEEE Int. Conf. on Robotics and Automation (ICRA2007), Rome, Italy, 2007, pp. 2928-2933.

[24] M. Grebenstein and P. van der Smagt, "Antagonism for a highly anthropomorphic hand-arm system," Advanced Robotics, vol. 22, pp. 39-55, 2008.

[25] S. Wolf and G. Hirzinger, "A New Variable Stiffness Design: Matching requirements of the next robot generation," IEEE Int. Conf. on Robotics and Automation (ICRA 2008), Pasadena, USA, 2008.

[26] S. Haddadin, T. Laue, U. Frese, and G. Hirzinger, "Foul 2050: Thoughts on Physical Interaction in Human-Robot Soccer," in IEEE/RSJ International Conference on Intelligent Robots and Systems (IROS2007), San Diego, USA, 2007, pp. 3243-3250.

\section{APPENDIX}

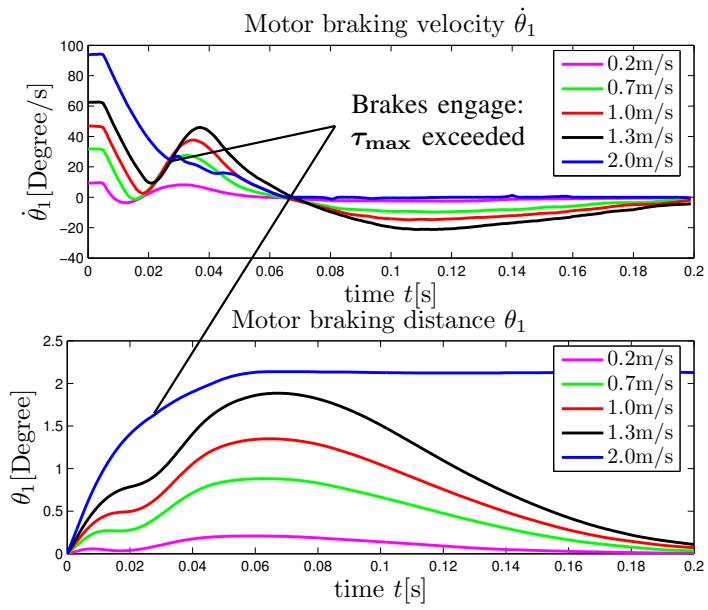

Fig. 8. Braking behavior of the motor in axis 1 for the LWRIII at various velocities. At $2 \mathrm{~m} / \mathrm{s}$ the maximal nominal joint torques are exceeded and a low-level safety feature causes the brakes to engage.

In Tab. I the braking distance of the LWRIII, resulting if impacting the mockup of a crash-test dummy head (dummydummy, see [1]), is shown to illustrate the effect external forces have on its braking distance. The robot's link side braking distance reduces by $>1 / 3$ with the given additional impact forces. The motor braking behavior and distance of the LWRIII can be extracted from Fig. 8, where the measured curve in absence of a collision is plotted. The motor reacts $4 \mathrm{~ms}$ after the stop is initialized, whereas the link side is delayed due to the intrinsic joint elasticities, see Fig. 9. The 

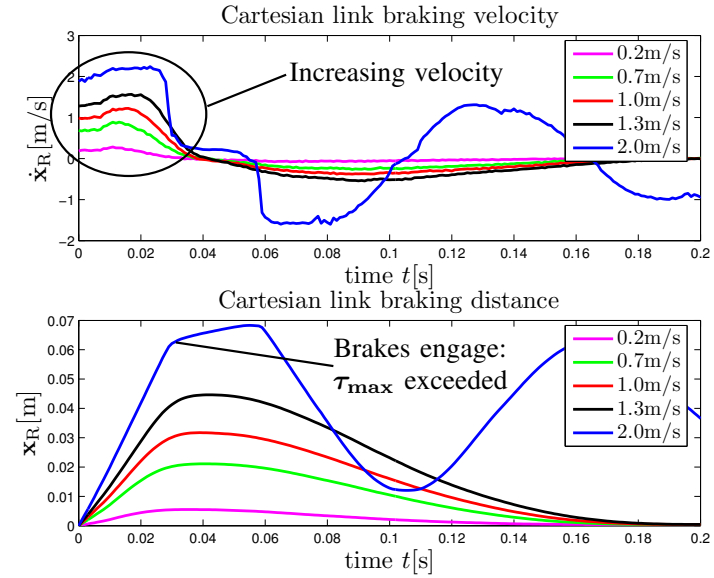

Fig. 9. Cartesian, i.e. link side braking behavior of the LWRIII at various velocities. At $2 \mathrm{~m} / \mathrm{s}$ the maximal nominal joint torques were exceeded, causing the robot to perform a low level stop engaging the brakes. Please note that the stop time is the same for all velocities. $\dot{\mathbf{x}}_{R}$ possibly increases in the beginning due to pretension in the joint springs and the lack of constant velocity phase.

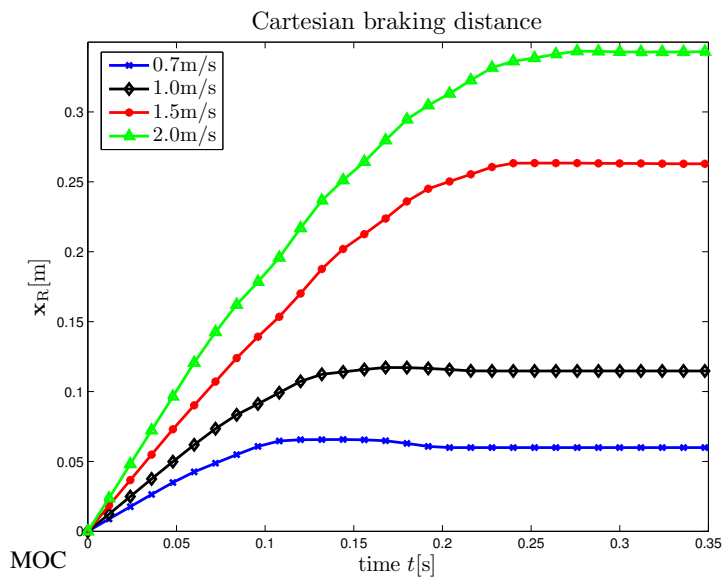

Fig. 10. Category 0 stop with collision for the $54 \mathrm{~kg}$ KUKA KR3-SI at various impact velocities. The braking distance is almost $5 \times$ the one of the LWRIII.

effect of increasing velocity is caused by the energy storage and release in the intrinsic joint spring ${ }^{20}$.

Braking distance and velocity profiles of the industrial robots are given in Fig. 10-12, where the point of origin $t=0$ indicates the beginning of physical contact with the dummydummy (MOC=Moment of contact). Because of their high inertias the industrial robots were not influenced noticeably by the impact with the dummy-dummy and therefore the results are, unlike for the LWRIII, not differentiated in Tab.I. This was confirmed by braking tests without external disturbances.

\footnotetext{
${ }^{20}$ In the next generation of our robots each joint will be equipped with adjustable mechanical stiffness [24], [25]. This mechanism can be used to store large amounts of potential energy and utilize them e.g. to achieve very high joint velocities as was already claimed in [26]. Apparently, already the moderate stiffness of the LWRIII can be used to show this effect.
}
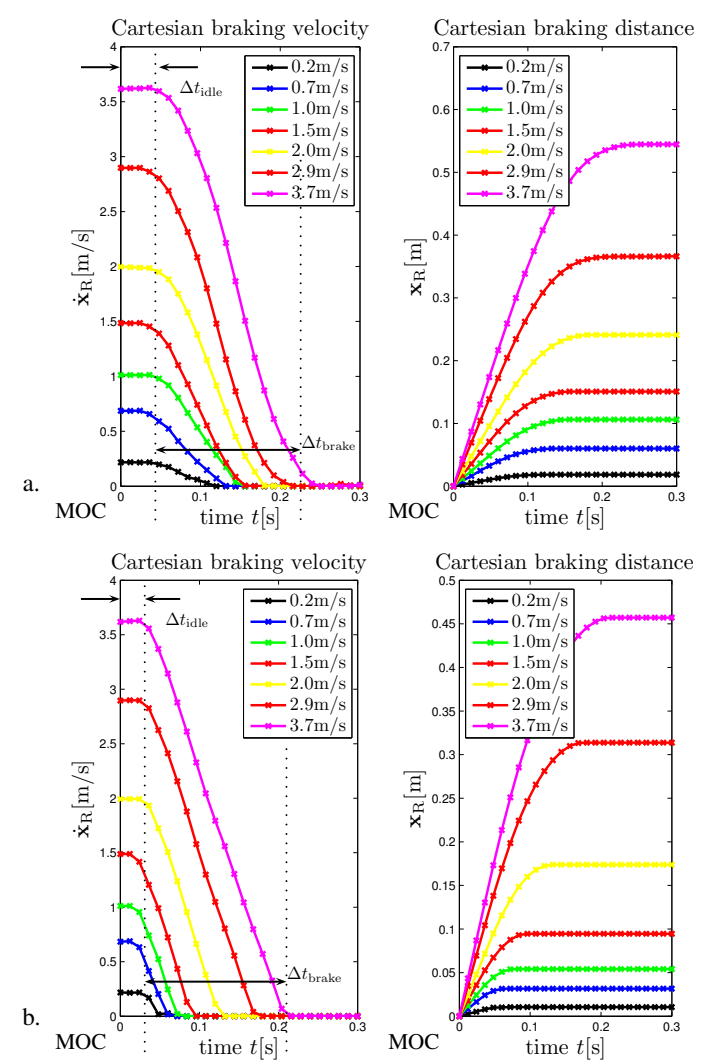

Fig. 11. Category 1 stop (a.) and Category 0 (b.) stop with collision for the $235 \mathrm{~kg}$ KUKA KR6 at various velocities up to maximal TCP velocities possible with joint 1 , i.e. $\dot{q}_{1}=\dot{q}_{1}^{\max }$. The idle and braking time at $3.7 \mathrm{~m} / \mathrm{s}$ are indicated.
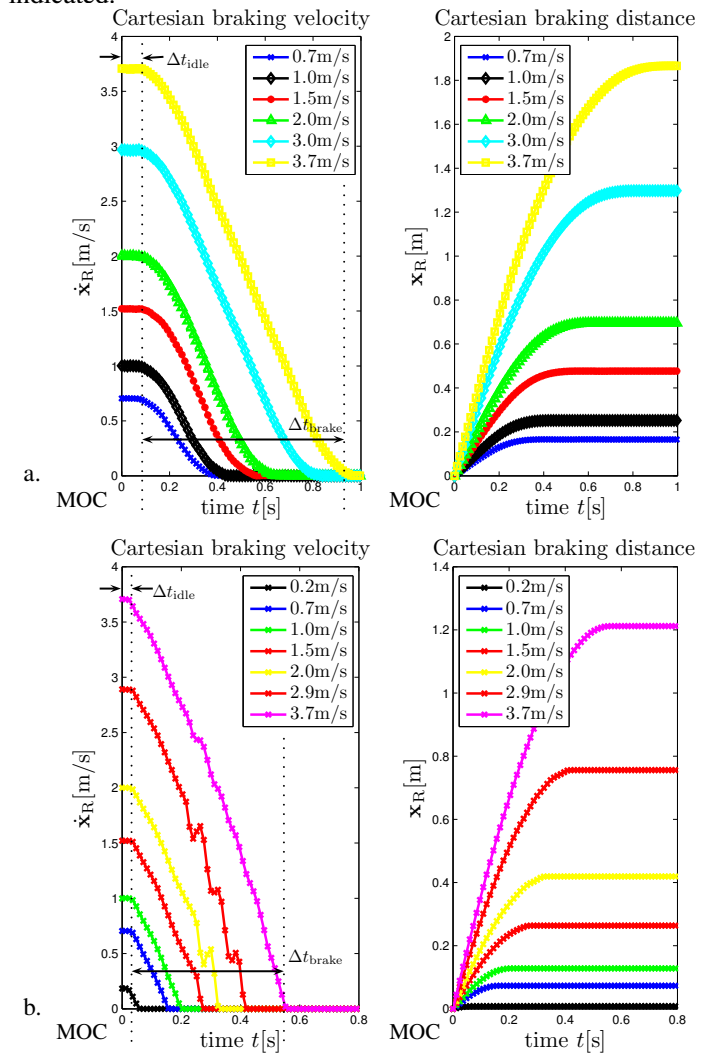

Fig. 12. Category 1 stop (a.) and Category 0 (b.) stop for the $2350 \mathrm{~kg}$ KUKA KR500 up to maximal TCP velocities possible with joint 1 , i.e. $\dot{q}_{1}=\dot{q}_{1}^{\max }$. The idle and braking time at $3.7 \mathrm{~m} / \mathrm{s}$ are indicated. 